\title{
Non-alcoholic compounds in beer have protective effects in Caco-2 cells by increasing the expression of genes in the Keap1-Nrf2 pathway
}

\author{
R. Watts, H. Lu, M. Arno, V.R. Preedy and P.A. Sharp \\ Diabetes \& Nutritional Sciences Division, King's College London, UK
}

Non-alcoholic compounds in beer, such as polyphenols, have been proposed to have beneficial effects on human health and moderate consumption is linked to a reduced risk of adverse chronic disease outcomes ${ }^{(1)}$. These protective effects are thought to occur via an upregulation of genes in cytoprotective pathways. We hypothesised that beer extract would upregulate genes in antioxidant pathways, contributing to a reduction of total oxidative stress. To address this we analysed expression of genes in human intestinal Caco- 2 cells following exposure to dealcoholised beer extract.

Caco-2 cells were seeded and prepared as previously described ${ }^{(2)}$. Cells were exposed to Dulbecco's modified Eagle medium containing degassed, dealcoholised freeze-dried beer extract for 24 hours. Control cells were exposed to medium alone. RNA was extracted from homogenised Caco-2 cells using TRIzol, and analysed via RNA probe target hybridisation using Affymetrix microarrays. Differences between beer extract-treated and control Caco-2 cells were indicated when there was a fold change in expression of $>50 \%$ in either direction, with a significance cut off of $p<0.05$. Data was analysed using Metacore GeneGo software which produced a list of significantly altered biological processes and pathways. To confirm the microarray results, RT-PCR was performed on those genes which were indicated as being significantly altered within the Keap1-Nrf2 pathway.

GeneGo analysis showed significant $(p<0 \cdot 001)$ alterations in the following oxidative stress pathways: 'Role of Sirtuin1 and PGC1 $\alpha$ in activation of antioxidant defence system', 'Activation of NOX1/2, DUOX1/2 NADPH oxidases' and 'Angiotensin II-induced production of ROS'. The expression of twelve genes within the Sirtuin1 \& PGC1 $\alpha$ antioxidant defence system were altered to a significant level $(p<0 \cdot 05)$. To confirm the microarray results, RT-PCR was performed on seven genes from the Keapl-Nrf2 pathway. Data from microarray and RT-PCR experiments were converted to fold changes of increased gene expression as to be directly comparable. The increases in fold expression from RT-PCR were: HMOX1 (+23.9), GCLreg (+7.5), GCLcat (+4.4), SQSTM1 (+3·3), KEAP1 (+2.4), SLC7A11 (+2.2), NQO1 (+2.1). All RT-PCR data for control versus beer extract were significant $p<0 \cdot 01$. The changes in NRF2 did not achieve significance. The microarray and RT-PCR results showed a positive correlation relationship $(p<0 \cdot 0001)$ of changes in expression in the seven Keap1-Nrf2 pathwaygenes.

These data suggest that the non-alcoholic compounds in beer increase expression of multiple genes in the Keapl-Nrf2 oxidative stress pathway. Although changes in Nrf2 itself were not significant, the pathway relies on an increase in 'free' rather than total $\mathrm{Nrf} 2^{(3)}$. Further research is required understand the molecular mechanisms involved in modulation of the Keapl-Nrf2 pathway before any practical or clinical application can be considered.

The authors would like to thank Dr Rukshana Hoque and Dr Stephanie Cheung for their assistance with cell culture and RNA extraction.

1. Ghiselli A, Natella F, Guidi A et al. (2000) J. Nutr. Biochem 11, 76-80.

2. Johnston K, Sharp P, Clifford M et al. (2005), FEBS Lett 579, 1653-1657.

3. Nguyen T, Nioi P \& Pickett CB (2009), J. Biol. Chem 284, 13291-13295 http://www.jbc.org/content/284/20/13291.full-target-1. 